\title{
A JORDAN DECOMPOSITION FOR OPERATORS IN BANACH SPACE $\left({ }^{1}\right)$
}

\author{
BY \\ SHMUEL KANTOROVITZ
}

Introduction. Let $X$ be a finite dimensional complex Euclidean space, and let $T$ be a linear operator acting on $X$. The Jordan decomposition theorem states that $T$ has a unique decomposition $T=S+N$, where $S=\int_{\sigma(T)} z d E(z), E$ is a spectral measure supported by the spectrum $\sigma(T)$ of $T$, and $N$ is a nilpotent operator commuting with $S$.

Our main result (Theorem 2.1) is a generalization of the Jordan theorem for operators with real spectrum to infinite dimensional reflexive Banach spaces. We consider operators $T$ satisfying the growth condition $\left|e^{i t T}\right|=O\left(|t|^{k}\right)$ for some integer $k \geqq 0$ and all real $t$. In $\S 1$, we construct the "Jordan manifold" for $T$, on which $T$ is shown to have a unique Jordan decomposition, if the spectrum (which is real because of the growth condition) has Lebesgue measure zero $(\$ 2)$. Related results are described in $\$ 2$. The theory is illustrated by examples in $\S 3$.

This work is clearly related to Dunford's theory of spectral operators. However, the latter is needed as a prerequisite only for Theorem 2.12. The standard reference is [1], [2].

Many thanks are due to Professors H. Furstenberg and C. A. McCarthy for discovering an error in the original version of this paper.

Notations. The following notations and conventions will be used throughout without further explanation: $X$ is an arbitrary complex Banach space. $B(X)$ denotes the Banach algebra of all bounded linear operators acting on $X$. Elements of $B(X)$ are simply called "operators." The identity operator is denoted by $I$. $X^{*}$ is the conjugate space of $X$. Norms are denoted by $|\cdot|$ (except when distinction is needed). The closure operation is denoted by an upper bar. For $T \in B(X)$, the symbols $\sigma(T), \rho(T), R(\lambda ; T)$ and $|T|_{\mathrm{sp}}$ stand respectively for the spectrum, the resolvent set, the resolvent, and the spectral norm of $T$. The restriction of $T$ to an invariant linear manifold $W$ is denoted by $T \mid W$. The real and complex fields are respectively denoted by $\boldsymbol{R}$ and $\boldsymbol{C}$. If $K \subset \boldsymbol{C}$ is compact, $C(K)$ denotes the Banach algebra of all continuous complex valued functions on $K$ with supremum norm. For $m=0,1,2, \cdots, C^{m}$ is the topological algebra of all complex-

Received by the editors May 24, 1965.

(1) Partly supported by NSF grant GP 780. 
valued functions on $\boldsymbol{R}$ with continuous derivatives up to the order $m$, with pointwise operations and with the topology of uniform convergence on every compact of all derivatives of order $\leqq m$. We let $C=C^{0}$ and $C_{0}^{m}=\left\{f \in C^{m} \mid f\right.$ has compact support $\}$. The Borel field of $R$ is denoted by $\mathscr{B}$.

1. The Jordan manifold. We recall first some of the terminology introduced in [7].

Definition 1.1. Let $m>0$ be an integer and $T \in B(X)$. A $C^{m}$-operational calculus for $T$ is a continuous representation $f \rightarrow T(f)$ of $C^{m}$ on $X$, such that (i) $T(1)=I$; (ii) $T(f)=T$ if $f(t) \equiv t$; and (iii) $T(\cdot)$ has compact support (denoted by $\Sigma=\Sigma(T)$ ). $\Sigma$ is defined as the smallest compact $K \subset R$ for which $T(f)=0$ whenever $f \in C^{m}$ has its support in the complement of $K . T$ is of class $C^{m}$ if there exists a $C^{m}$-operational calculus for $T$. In this case, the latter is unique, and $\Sigma=\sigma(T)$ (cf. Lemmas 2.2 and 2.8 in [7] or Proposition 1 in [5]).

Let $T \in B(X)$ be such that

$$
\left|e^{i t T}\right|=O\left(|t|^{k}\right), \quad t \in R,|t| \rightarrow \infty
$$

for some integer $k \geqq 0$.

By Lemma 2.11 in [7], $T$ has real spectrum and is of class $C^{m}$ for $m \geqq k+2$.

Definition 1.2. Let $T \in B(X)$ satisfy (1.1), fix $m \geqq k+2$, and let $T(\cdot)$ be the $C^{m}$-operational calculus for $T$. We write

1. $|f|_{m, T}=\sum_{j=0}^{m}(1 / j !) \max _{\sigma(T)}\left|f^{(j)}\right|, f \in C^{m}$;

2. $|x|_{m, T}=\sup \left\{|T(f)| ; f \in C^{m},|f|_{m, T} \leqq 1\right\}$;

3. $D_{m}=\left\{x \in X ;|x|_{m, T}<\infty\right\}=D_{m}(T)$;

4. $D=\bigcup_{m \geqq k+2} D_{m}=D(T)$.

We call $D$ the Jordan manifold for $T$.

The function $x \rightarrow|x|_{m, T}$ is homogeneous and subadditive for each fixed $m \geqq k+2$. Therefore $D_{m}$ is a linear manifold. For $x$ fixed, $|x|_{m, T}$ is a nonincreasing function of $m(m \geqq k+2)$. Therefore

$$
D_{k+2} \subseteq D_{k+3} \subseteq \cdots
$$

and $D$ is a linear manifold.

Fix $m \geqq k+2$. Since $T(1)=I,|x|_{m, T} \geqq|x|$ for all $x \in X$; therefore $|x|_{m, T}$ is a norm on $D_{m}$.

If $V \in B(X)$ commutes with $T$, it commutes with $T(f)$ for all $f \in C^{m}$. Hence, for all $x \in D_{m}$ and $f \in C^{m}$ with $|f|_{m, T} \leqq 1$, we have

$$
|T(f) V x|=|V T(f) x| \leqq|V||T(f) x| \leqq|V||x|_{m, T},
$$

i.e., $V x \in D_{m}$ and $|V x|_{m, T} \leqq|V||x|_{m, T}$. Thus $D_{m}$ is an invariant linear manifold for $V$, and $V$ is continuous on the normed linear space $\left(D_{m},|\cdot|_{m, T}\right)$. These facts are valid in particular for $V=T(f)$ for each $f \in C^{k+2}$. For $x \in D_{m}$ fixed, consider 
the linear map $f \rightarrow T(f) x$ of $C^{m}$ into $\left(D_{m},|\cdot|_{m, T}\right)$. When $C^{m}$ is endowed with the pseudo-norm $|f|_{m, T}$, this map is continuous with bound $\leqq|x|_{m, T}$, i.e.,

$$
|T(f) x|_{m, T} \leqq|x|_{m, T} \cdot|f|_{m, T}
$$

for all $f \in C^{m}$ and $x \in D_{m}$. Indeed,

$$
\begin{aligned}
|T(f) x|_{m, T} & =\sup \left\{|T(g f) x| ; g \in C^{m},|g|_{m, T} \leqq 1\right\} \\
& \leqq \sup \left\{|T(h) x| ; h \in C^{m},|h|_{m, T} \leqq|f|_{m, T}\right\} \\
& \leqq|x|_{m, T} \cdot|f|_{m, T} .
\end{aligned}
$$

If $D$ is of the second category in $X$, then $D=D_{m}=X$ for some $m \geqq k+2$, by the uniform boundedness theorem; the norms $|x|$ and $|x|_{m, T}$ are then equivalent, by the closed graph theorem. We shall see later on that $D \neq X$ in general. However, it follows easily from [10, pp. 99-100] that there exists an $m \geqq k+2$ such that $D=D_{m}=X$, provided that $\sigma(T)$ is a finite union of points and closed intervals. Of this particular situation, only the following is relevant to our subsequent analysis.

Proposition 1.1. If $\sigma(T)$ is a finite point set, then $D=D_{k+2}=X$.

Proof. For each $x \in X$ and $x^{*} \in X^{*}, x^{*} T(\cdot) x$ is a distribution of order $\leqq k+2$ with support $\sigma(T)=\left\{p_{1}, \cdots, p_{n}\right\}$. By [10, pp. 99-100], there exist $M>0$ and $C_{i j}=C_{i j}\left(x, x^{*}\right) \in C \quad(i=1, \cdots, n ; j=0, \cdots, k+2)$ such that $\left|C_{i j}\left(x, x^{*}\right)\right| \leqq M|x|\left|x^{*}\right|$ and $x^{*} T(f) x=\sum_{i, j} C_{i j} f^{(j)}\left(p_{i}\right)$ for all $f \in C^{k+2}, x \in X$ and $x^{*} \in X^{*}$. Therefore $|T(f) x| \leqq M^{\prime}|x||f|_{k+2, T}$ for all $f \in C^{k+2}$, and $x \in X$, where $M^{\prime}>0$ depends only on $T$.

COROllary 1.2. If $W$ is a finite dimensional invariant subspace for $T$, then $W \subset D_{k+2}$.

Proof. Since $T \mid W$ has finite spectrum and satisfies (1.1), $D_{k+2}(T \mid W)=W$ by Proposition 1.1. But $D_{k+2}(T \mid W)=D_{k+2}(T) \cap W$. Hence $W \subset D_{k+2}(T)$.

COROLlaRy 1.3. $D_{k+2}$ contains all the eigenvectors of $T$ (therefore, if the eigenvectors of $T$ are fundamental in $X, D_{k+2}$ (and hence $\left.D\right)$ is dense in $\left.X\right)$.

Corollary 1.4. Let $\lambda$ be an isolated point of $\sigma(T)$, and let $E(\lambda)$ be the associated projection (cf. [3, Volume I, p. 573]). Then $E(\lambda) X \subset D_{k+2}$.

Proof. Since $\sigma(T \mid E(\lambda) X)=\{\lambda\}$ (Theorem VII, 3.20 in [3]),

$$
E(\lambda) X=D_{k+2}(T \mid E(\lambda) X) \subset D_{k+2}(T) .
$$

Thus $D_{k+2} \neq 0$ if there are isolated points in the spectrum. More can be said if all nonzero points in the spectrum are isolated (hence, in particular, if $T$ is compact). 
THEOREM 1.5. Let $T \in B(X)$ satisfy (1.1) Suppose that all nonzero points in $\sigma(T)$ are isolated. Then $\bar{D}_{k+2}$ contains the closed range of $T^{k+1}$. If $T$ satisfies (1.1) with $k=0$ and if $X$ is reflexive, then $D_{k+2}$ is dense in $X$.

We state first two lemmas, which are implicit in the proofs of Theorem XI, 6.29 and Corollary XI, 6.30 in [3, Volume II]. Let $Z$ be the closed linear span of $\{E(\lambda) X: \lambda \in \sigma(T)-\{0\}\}$, and let $Z^{\perp}$ be the annihilator of $Z$ in $X^{*}$.

LEMMA 1.6. Suppose all the nonzero points in the spectrum of an operator $T \in B(X)$ are isolated. Then $T^{*} \mid Z^{\perp}$ is quasi-nilpotent.

Lemma 1.7. Let $X$ be reflexive and $T \in B(X)$. Suppose that $\lambda_{n} R\left(\lambda_{n} ; T\right)$ is bounded for some sequence $\left\{\lambda_{n}\right\} \subset \rho(T)$ converging to 0 . Then

$$
\overline{\mathscr{R}}+\mathscr{N}=X \text {, }
$$

where $\mathscr{R}$ and $\mathscr{N}$ are the range and null space of $T$ respectively.

We need also a theorem of Naimark's [8, p. 208] in the following form:

LEMMA 1.8 (NAIMARK). Let $T$ be a quasi-nilpotent element of a normed algebra $R$. Suppose that for some integer $n \geqq 1$

$$
\left|\lambda^{n} R(\lambda ; T)\right|=O\left((\sin \theta)^{-n}\right)
$$

for $\theta=\arg \lambda \neq 0, \pi$. Then $T^{n}=0$.

The next lemma puts Condition (1.1) in the form (1.3).

Lemma 1.9. If $T \in B(X)$ satisfies (1.1), then

$$
\left|\lambda^{k+1} R(\lambda ; T)\right|=O\left((\sin \theta)^{-(k+1)}\right) \text { for } \theta=\arg \lambda \neq 0, \pi \text {. }
$$

Proof. Condition (1.1) implies that

$$
\begin{aligned}
R(\lambda ; T) & =i \int_{0}^{\infty} e^{-i \lambda t} e^{i t T} d t \quad \text { for } \operatorname{Im} \lambda<0 \\
& =-i \int_{0}^{\infty} e^{i \lambda t} e^{-i t T} d t \quad \text { for } \operatorname{Im} \lambda>0
\end{aligned}
$$

where the integrals converge in $B(X)$.

Hence, for $\eta<0$,

$$
\begin{aligned}
|R(\xi+i \eta ; T)| & \leqq \int_{0}^{\infty} e^{\eta t}\left|e^{i t T}\right| d t=O\left(\int_{0}^{\infty} e^{\eta t} t^{k} d t\right) \\
& =O\left(|\eta|^{-(k+1)}\right)
\end{aligned}
$$

and similarly for $\eta>0$.

Thus $|R(\lambda ; T)|=O\left((\operatorname{Im} \lambda)^{-(k-1)}\right)$ for $\operatorname{Im} \lambda \neq 0$, which is equivalent to (1.4). 
Proof of Theorem 1.5. Consider the operator $T^{*} \mid Z^{\perp}$. It is quasi-nilpotent (Lemma 1.6) and satisfies (1.3) with $n=k+1$ (by Lemma 1.9, since $\left.\left|R\left(\lambda ; T^{*} \mid Z^{\perp}\right)\right| \leqq\left|R\left(\lambda ; T^{*}\right)\right|=|R(\lambda ; T)|\right)$. Hence, by Lemma $1.8, T^{* k+1} x^{*}=0$ for all $x^{*} \in Z^{\perp}$, i.e., $x^{*} T^{k+1} x=0$ for all $x \in X$ and $x^{*} \in Z^{\perp}$. By Hahn-Banach, this implies that $Z \supset \overline{T^{k+1} X}$. But $\bar{D}_{k+2} \supset Z$ by Corollary 1.4. Hence $\bar{D}_{k+2}$ contains the closed range of $T^{k+1}$.

In particular, if $T$ satisfies (1.1) with $k=0, \bar{D}_{k+2} \supset \overline{\mathscr{R}}$. Since $D_{k+2} \supset \mathscr{N}$ trivially, we conclude that $\bar{D}_{k+2}=X$ if $X$ is reflexive by applying Lemma 1.7.

Recall that $x \in X$ is called a root vector of $T$ if $(\lambda I-T)^{n} x=0$ for some $\lambda \in C$ and some integer $n \geqq 1$.

Proposition 1.10. Let $T \in B(X)$ satisfy (1.1). Then its Jordan manifold contains the linear span of all the root vectors of $T$.

Proof. It suffices to show that the null space of $(\lambda I-T)^{n}$ is contained in $D$, for all $\lambda \in \sigma(T)$ and all integers $n \geqq 1$.

Let $S=T-\lambda I$ for $\lambda \in \sigma(T)$ fixed. Since $\lambda$ is real, $S$ satisfies (1.1) and $D_{m}(S)=D_{m}(T)$ for each $m \geqq k+2$. Hence it suffices to show that if $x \neq 0$ satisfies $S^{n} x=0$ for some $n \geqq 1$, then $x \in D(S)$.

Let $m \geqq \max \{k+2, n-1\}$. Clearly, $|x|_{m, S}=\sup \left\{|S(f) x|: f \in C_{0}^{m},|f|_{m, S} \leqq 1\right\}$. For $f \in C_{0}^{m}, S(f)$ is given by

$$
S(f)=\int_{R} \hat{f}(t) e^{i t s} d t
$$

where $f(t)=(1 / 2 \pi) \int_{R} e^{-i t s} f(s) d s$ and the integral in (1.5) converges in $B(X)$ (cf. proof of Lemma 2.11 in [7]). For $x$ as above,

$$
\begin{aligned}
S(f) x & =\int_{R} \hat{f}(t) \sum_{j=0}^{n-1} \frac{(i t)^{j}}{j !} S^{j} x d t \\
& =\sum_{j=0}^{n-1} \frac{1}{j !} \int_{R} \hat{f}(t)(i t)^{j} d t S^{j} x \\
& =\sum_{j=0}^{n-1} \frac{f^{(j)}(0)}{j !} S^{j} x .
\end{aligned}
$$

Since $m \geqq n-1$ and $0 \in \sigma(S)$, we conclude that

$$
|x|_{m, s} \leqq \max _{0 \leqq j \leqq n-1}\left|S^{j} x\right|<\infty, \quad \text { i.e., } x \in D_{m}(S) \subset D .
$$

2. The Jordan decomposition. In order to avoid repetition, we state the following

STANDING HYPOTHESIS.

(a) $X$ is a reflexive Banach space. 
(b) $T \in B(X)$ satisfies Condition (1.1).

(c) $\sigma(T)$ (which is a subset of $\boldsymbol{R}$ by (1.1)) has linear Lebesgue measure zero. We follow the notations of $\S 1$ without further explanation. It is also convenient to introduce the following:

Definition 2.1. Let $W \subset X$ be a linear manifold. We denote by $\boldsymbol{T}(W)$ the algebra of all linear transformations of $X$ with domain $W$ and range contained in $W$.

A generalized spectral measure on $W$ is a map $\delta \rightarrow E(\delta)$ of $\mathscr{B}$ into $T(W)$ such that

(i) $E(R) x=x$ for all $x \in W$, and

(ii) $E(\cdot) x$ is a bounded regular strongly countably additive vector measure on $\mathscr{B}$, for each $x \in W$.

Notice that a vector measure $\mu$ is "regular"' if $x^{*} \mu$ is a regular complex measure for all $x^{*} \in X^{*}$.

We can state now our main result, which is a generalization of the classical Jordan decomposition theorem for complex matrices with real spectrum to infinite dimensional Banach spaces.

THEOREM 2.1. There exist $S, N \in T(D)$ such that

(2.1) $T \mid D=S+N$,

(2.2) $S N=N S$,

(2.3) $N^{k+1}=0$, and

(2.4) $p(S) x=\int_{\sigma(T)} p(t) d E(t) x, x \in D$

for all polynomials $p$, where $E(\cdot)$ is a generalized spectral measure on $D$ supported by $\sigma(T)$ and commuting with any $V \in B(X)$ which commutes with $T$.

This decomposition is "maximal-unique", meaning that if $W$ is an invariant linear manifold for $T$ for which (2.1)-(2.4) are valid with $W$ instead of $D$, then $W \subset D$ and the transformations $S, N$ and $E(\delta)(\delta \in \mathscr{B})$ corresponding to $W$ are the restrictions to $W$ of the respective transformations associated with $D$.

Finally, $D=D_{k+2}$ and for each $x \in D$, the map $f \rightarrow T(f) x$ of $C^{k+2}$ into $X$ has an extension as a continuous linear map of $C^{k}$ into $D$ given by

$$
T(f) x=\sum_{j=0}^{k} \frac{1}{j !} \int_{\sigma(T)} f^{(j)}(t) d E(t) N^{j} x
$$

(for all $f \in C^{k}$ and each $x \in D$ ). The extended map $f \rightarrow T(f)$ of $C^{k}$ into $T(D)$ is multiplicative.

The proof is organized in a series of lemmas.

LEMMA 2.2. There exists a uniquely determined family

$$
\left\{E_{j, m}(\delta) ; m \geqq k+2 ; j=0, \cdots, m ; \delta \in \mathscr{B}\right\}
$$

of linear transformations of $X$ with the following properties:

(a) $E_{j, m}(\delta)$ has domain $D_{m}$; 
(b) for each $x \in D_{m}, E_{j, m}(\cdot) x$ is a regular, uniformly bounded, strongly countably additive vector measure supported by $\sigma(T)$; and

$$
T(f) x=\sum_{j=0}^{m} \frac{1}{j !} \int_{\sigma(T)} f^{(j)}(t) d E_{j m}(t) x
$$

for all $x \in D_{m}$ and $f \in C^{m}$.

Proof. Let $C(\sigma(T))^{m+1}(m=0,1, \cdots)$ be the Cartesian product of $m+1$ copies of $C(\sigma(T))$. Its elements are denoted by $\boldsymbol{f}=\left(f_{0}, f_{1}, \cdots, f_{m}\right)$, and it is normed by

$$
|f|=\sum_{j=0}^{m} \frac{1}{j !} \sup _{\sigma(T)}\left|f_{j}\right|, \quad f \in C(\sigma(T))^{m+1} .
$$

Let $A_{m}$ be the linear manifold in $C(\sigma(T))^{m+1}$ consisting of all $f=\left(f_{0}, f_{1}, \cdots, f_{m}\right)$ with $f_{j}=f^{(j)} \mid \sigma(T)$ for some $f \in C^{m}(j=0, \cdots, m)$. If $f \in A_{m}$ and $f$ is any element of $C^{m}$ such that $f_{j}=f^{(j)} \mid \sigma(T), j=0, \cdots, m$, then $|f|=|f|_{m, T}$. Fix $m \geqq k+2$, $x \in D_{m}$ and $x^{*} \in X^{*}$.

Consider the map

$$
\pi_{m}\left(x, x^{*}\right): f \in A_{m} \rightarrow x^{*} T(f) x
$$

where $f$ is any function in $C^{m}$ such that $f_{j}=f^{(j)} \mid \sigma(T)$ for all $j=0, \cdots, m$.

Since $x \in D_{m}$, this map is well defined, linear and continuous, because by (1.2),

$$
\begin{aligned}
\left|\pi_{m}\left(x, x^{*}\right) f\right| & =\left|x^{*} T(f) x\right| \leqq\left|x^{*}\right||T(f) x| \leqq\left|x^{*}\right||T(f) x|_{m, T} \\
& \leqq\left|x^{*}\right||x|_{m, T}|f|_{m, T}=\left|x^{*}\right||x|_{m, T}|f|
\end{aligned}
$$

By the Hahn-Banach and the Riesz representation theorems, there exist $m+1$ regular finite Borel measures $\mu_{j, m}=\mu_{j, m}\left(\cdot \mid x, x^{*}\right)(j=0, \cdots, m)$ on $\boldsymbol{R}$, which are supported by $\sigma(T)$ and satisfy

$$
\begin{gathered}
\operatorname{var} \mu_{j, m}\left(\cdot \mid x, x^{*}\right) \leqq\left|x^{*}\right||x|_{m, T} \quad(j=0, \cdots, m), \text { and } \\
\pi_{m}\left(x, x^{*}\right) f=\sum_{j=0}^{m} \frac{1}{j !} \int_{\sigma(T)} f^{(j)} d \mu_{j, m} \quad \text { for all } f \in A_{m} .
\end{gathered}
$$

Since $\sigma(T)$ is a Lebesgue null set, the measures $\mu_{j, m}$ are singular with respect to the Lebesgue measure on $\boldsymbol{R}$. Therefore, by Lemma 3.7 in [7], the representation (2.6) is unique. For $x \in D_{m}$ fixed and $x^{*}$ varying in $X^{*}$, the linearity of $\pi_{m}\left(x, x^{*}\right) f=x^{*} T(f) x$ in $x^{*}$ and the uniqueness of the representation (2.6) imply the linearity of $\mu_{j, m}\left(\delta \mid x, x^{*}\right)$ in $x^{*}$ for each fixed $\delta \in \mathscr{B}$ and $j=0, \cdots, m$. Since $X$ is reflexive, there exists a unique element of $X$ (which we denote by $\left.E_{j, m}(\delta) x\right)$, with norm $\leqq|x|_{m, T}$, such that $\mu_{j, m}\left(\delta \mid x, x^{*}\right)=x^{*} E_{j, m}(\delta) x$ for all $x^{*} \in X^{*}(j=0, \cdots, m ; \delta \in \mathscr{B})$. The uniqueness of the representation (2.6) implies that $E_{j, m}(\delta)$ is a linear tranformation of $X$ with domain $D_{m}$ (for each $\delta \in \mathscr{B}$ and 
$j=0, \cdots, m)$. Now (b) follows from the Orlicz-Banach-Pettis theorem and (c) is a rewriting of (2.6).

LEMMA 2.3. If $V \in B(X)$ commutes with $T$, then $V$ commutes with $E_{j, m}(\delta)$ for all $\delta \in \mathscr{B}, m \geqq k+2$ and $j=0, \cdots, m$.

Proof. Fix $m \geqq k+2$. If $V$ commutes with $T$, then it commutes with $T(f)$ for each $f \in C^{m}$ and $V D_{m} \subset D_{m}$ (cf. $\$ 1$ ).

Therefore by Lemma 2.2 (c)

$$
\begin{aligned}
V T(f) x & =\sum_{j=0}^{m} \frac{1}{j !} \int_{\sigma(T)} f^{(j)}(t) d V E_{j, m}(t) x \\
& =T(f) V x \\
& =\sum_{j=0}^{m} \frac{1}{j !} \int_{\sigma(T)} f^{(j)}(t) d E_{j, m}(t) V x
\end{aligned}
$$

for all $x \in D_{m}$ and $f \in C^{m}$, and the lemma follows from Lemma 3.7 in [7], because all the measures involved are singular with respect to the Lebesgue measure on $\boldsymbol{R}$.

Let $\boldsymbol{B}(\sigma(T))$ denote the space of all complex Borel functions $f$ on $\boldsymbol{R}$, which are bounded on $\sigma(T)$, with the pseudo-norm $\sup _{\sigma(T)}|f|$.

In the next lemma, we consider $D_{m}$ as a normed linear space with the norm $|x|_{m, T}$.

LEMMA 2.4. Fix $m \geqq k+2$ and $0 \leqq j \leqq m$. Consider the map $\phi_{j, m}$ of $\boldsymbol{B}(\sigma(T)) \times D_{m}$ into $X$ defined by

$$
\phi_{j, m}:(f, x) \rightarrow \int_{\sigma(T)} f(t) d E_{j, m}(t) x .
$$

Then $\phi_{j, m}$ is continuous, has range in $D_{m}$, and is also continuous as a map of $\boldsymbol{B}(\sigma(T)) \times D_{m}$ into $D_{m}$. In fact

$$
\left|\int_{\sigma(T)} f(t) d E_{j, m}(t) x\right|_{m, T} \leqq \sup _{\sigma(T)}|f| \cdot|x|_{m, T},
$$

for all $f \in \boldsymbol{B}(\sigma(T)), x \in D_{m}$ and $j=0, \cdots, m$.

Proof. Let $(f, x) \in B(\sigma(T)) \times D_{m}$. Then $y=\phi_{j, m}(f, x)$ is a well-defined element of $X$, by Lemma 2.2 (b). For $g \in C^{m}$,

$$
T(g) y=\int_{\sigma(T)} f(t) d T(g) E_{j, m}(t) x=\int_{\sigma(T)} f(t) d E_{j, m}(t) T(g) x,
$$

by Lemma 2.3 .

Since $T(g) x \in D_{m}$, we have 


$$
x^{*} E_{j, m}(\cdot) T(g) x=\mu_{J, m}\left(\cdot \mid T(g) x, x^{*}\right)
$$

for all $x^{*} \in X^{*}$. Therefore, by (1.2) and (2.6),

$$
\begin{aligned}
\left|x^{*} T(g) y\right| & \leqq \sup _{\sigma(T)}|f| \cdot \operatorname{var} \mu_{j, m}\left(\cdot \mid T(g) x, x^{*}\right) \\
& \leqq \sup _{\sigma(T)}|f| \cdot\left|x^{*}\right||T(g) x|_{m, T} \\
& \leqq \sup _{\sigma(T)}|f| \cdot\left|x^{*}\right||x|_{m, T}|g|_{m, T} .
\end{aligned}
$$

Hence

$$
|y|_{m, T}=\sup _{\left|x^{*}\right|=1} \sup _{|g|_{m}, T \leqq 1}\left|x^{*} T(g) y\right| \leqq \sup _{\sigma(T)}|f||x|_{m, T},
$$

proving (2.7) and the lemma (since $|y| \leqq|y|_{m, T}$ ).

Corollary 2.5. For $m \geqq k+2, j=0, \cdots, m$ and $\delta \in \mathscr{B}$

In fact,

$$
E_{j, m}(\delta) D_{m} \subset D_{m}
$$

$$
\left|E_{j, m}(\delta) x\right|_{m, T} \leqq|x|_{m, T} \text { for all } x \in D_{m} .
$$

Taking $f=1$ in Lemma $2.2(\mathrm{c})$, we see that $E_{0, m}(R) x=x$ for all $x \in D_{m}$. Together with Lemma 2.2, Lemma 2.3 and Corollary 2.5, this gives

COROLlARY 2.6. For each $m \geqq k+2, E_{m}=E_{0, m}$ is a generalized spectral measure on $D_{m}$, supported by $\sigma(T)$ and commuting with any $V \in B(X)$ which commutes with $T$.

Next, take $f(t) \equiv t$ in Lemma 2.2(c): we obtain

$$
T x=\int_{\sigma(T)} t d E_{0, m}(t) x+E_{1, m}(R) x \quad \text { for all } x \in D_{m} .
$$

Let $S_{m}$ and $N_{m}$ be the transformations defined on $D_{m}$ by the first and second terms on the right-hand side of (2.8), respectively.

Then $T \mid D_{m}=S_{m}+N_{m}$, and $S_{m}, N_{m} \in T\left(D_{m}\right)$ by Lemma 2.4. We proceed to show that $S_{m}$ and $N_{m}$ have the properties (2.2)-(2.4). The crucial step consists in proving an identity relating the Fourier-Stieltjes transforms of the vector measures $E_{j, m}(\cdot) x\left(m \geqq k+2,0 \leqq j \leqq m, x \in D_{m}\right)$.

DEFINITION 2.2. For $m \geqq k+2$ and $0 \leqq j \leqq m$, let

$$
E_{j, m}(s) x=\int_{o(T)} e^{i t s} d E_{j, m}(t) x, \quad s \in R, x \in D_{m} .
$$

By Lemma $2.4, E_{j, m}(s)$ is a well-defined element of $T\left(D_{m}\right)$, for each $j=0, \cdots, m$ and $s \in \boldsymbol{R}$.

LeMma 2.7. Fix $m \geqq k+2$. Then, for all $j, h=0, \cdots, m$ and $s, u \in R$, 


$$
\begin{aligned}
E_{j, \hat{m}}(s) E_{h, \hat{m}}(u) & =E_{j+h, \hat{m}}(s+u) & & \text { if } j+h \leqq m, \\
& =0 & & \text { if } j+h>m .
\end{aligned}
$$

Proof. For $s \in \boldsymbol{R}$ fixed, take $f_{s}(t)=e^{i s t}$ in Lemma 2.2(c):

$$
e^{i s T} x=\sum_{j=0}^{m} \frac{(i s)^{j}}{j !} E_{j, m}(s) x, \quad s \in R, x \in D_{m} .
$$

Using (2.9) in the identity $e^{i(s+u) T} x=e^{i s T}\left(e^{i u T} x\right)$ and recalling that $e^{i u T} x \in D_{m}$ for $x \in D_{m}$, we obtain after some rearrangement:

$$
\begin{aligned}
\sum_{h=0}^{m}(i s)^{h} \int_{\sigma(T)} e^{i s t} e^{i u t} d_{t}\left\{\frac{1}{h !} \sum_{j=0}^{m-h} \frac{(i u)^{j}}{j !} E_{j+h, m}(t) x\right\} \\
=\sum_{h=0}^{m}(i s)^{h} \int_{\sigma(T)} e^{i s t} d_{t}\left\{\frac{1}{h !} E_{h, m}(t) e^{i u T} x\right\}
\end{aligned}
$$

for all $s, u \in R$ and $x \in D_{m}$ (the subscript $t$ of $d$ indicates that integration is performed with respect to $t$ ).

Since $\sigma(T)$ has Lebesgue measure zero, it follows from Lemma 3.11 in [7] (applied with $u$ fixed and $s$ variable) that

$$
\int_{\sigma(T)} e^{i s t} e^{i u t} d_{t}\left\{\sum_{j=0}^{m-h} \frac{(i u)^{j}}{j !} E_{j+h, m}(t) x\right\}=\int_{\sigma(T)} e^{i s t} d_{t}\left\{E_{h, m}(t) e^{i u T} x\right\}
$$

for all $s, u \in R$ and $x \in D_{m}$.

By Lemma 2.3 , by the fact that $E_{j, m}(s)$ has range contained in $D_{m}$, and by (2.9), we obtain

$$
\begin{aligned}
\sum_{j=0}^{m-h} \frac{(i u)^{j}}{j !} \int_{\sigma(t)} e^{i u t} e^{i s t} d E_{j+h, m}(t) x & =E_{h, m}(s) e^{i u T} x \\
& =e^{i u T} E_{h, \hat{m}}(s) x \\
& =\sum_{j=0}^{m} \frac{(i u)^{j}}{j !} \int_{\sigma(T)} e^{i u t} d E_{j, m}(t) E_{h, \hat{m}}(s) x
\end{aligned}
$$

for all $s, u \in R$ and $x \in D_{m}$.

Applying Lemma 3.11 in [7] (with $u$ variable and $s$ fixed), the result follows. Definition 2.3. Fix $m \geqq k+2$. For $f \in B(\sigma(T))$, let $S_{m}(f) \in T\left(D_{m}\right)$ be defined by

$$
S_{m}(f) x=\int_{\sigma(T)} f(t) d E_{m}(t) x, \quad x \in D_{m}
$$

(cf. Lemma 2.4 and Corollary 2.6).

LEMMA 2.8. The map $f \rightarrow S_{m}(f)$ of $C(\sigma(T))$ into $T\left(D_{m}\right)$ is multiplicative. 
Proof. By Lemma 2.7 with $j=h=0$, we have

$$
\hat{E_{m}}(s) \hat{E_{m}}(u)=\hat{E_{m}}(s+u) \text { for all } s, u \in R \text {. }
$$

It follows that the map $f \rightarrow S_{m}(f)$ is multiplicative when restricted to functions $f$ of the form

$$
f(t)=\sum_{j=1}^{N} C_{j} \exp \left(i s_{j} t\right) \quad\left(C_{j} \in C ; t, s_{j} \in R\right) .
$$

Since these functions are dense in $C(\sigma(T))$, the result follows easily from Lemma 2.4 (cf. inequality (2.7)).

LEMMA 2.9. Fix $m \geqq k+2$. Then

(i) $N_{m}$ commutes with $S_{m}(f)$ for all $f \in C(\sigma(T))$;

(ii) $E_{j, m}(\delta)=E_{m}(\delta) N_{m}^{j}$ for all $\delta \in \mathscr{B}$ and $j=1, \cdots, m$.

(iii) $N_{m}^{k+1}=0$.

Proof. For $1 \leqq r \leqq m$, take $j=r-1$ and $h=1$ in Lemma 2.7; for $u=0$, we obtain

$$
E_{r, m}(s)=E_{r-1, \hat{m}}(s) N_{m},
$$

hence

$$
E_{r, m}(s)=\hat{E_{m}}(s) N_{m}^{r} \quad(1 \leqq r \leqq m, s \in R) .
$$

Interchanging the roles of $j$ and $h$, we obtain similarly

$$
E_{r, m}(s)=N_{m}^{r} E_{m}^{\hat{m}}(s) \quad(1 \leqq r \leqq m, s \in R) .
$$

By (2.10) and (2.11), $N_{m}$ commutes with $S_{m}(f)$ for functions $f$ of the form

$$
f(t)=\sum_{j=1}^{N} C_{j} \exp \left(i s_{j} t\right) \quad\left(C_{j} \in C, s_{j} \in R\right),
$$

and (i) follows by a density argument as in Lemma 2.8, using (2.7).

(ii) follows from (2.10) and the uniqueness theorem for Fourier-Stieltjes transforms of regular Borel measures.

By (2.9) and (2.11),

$$
e^{i s T} x=\sum_{j=0}^{m} \frac{(i s)^{j}}{j !} N_{m}^{j} E_{m}^{\wedge}(s) x, \quad s \in R, \quad x \in D_{m}
$$

( $N_{m}^{0}$ stands for $\left.I\right)$.

Since $E_{m}^{\hat{m}}(s) \hat{E_{m}}(-s)=x$ by Lemma 2.7 , we have

$$
e^{i s T} E_{m}^{\wedge}(-s) x=\sum_{j=0}^{m} \frac{(i s)^{j}}{j !} N_{m}^{j} x, \quad s \in R, x \in D_{m} .
$$


Fix $x \in D_{m}$. By (2.7),

$$
\left|e^{i s T} E_{m}(-s) x\right| \leqq\left|e^{i s T}\right|\left|E_{m}^{\hat{m}}(-s) x\right| \leqq\left|e^{i s T}\right||x|_{m, T}=O\left(|s|^{k}\right)
$$

for $s \in \boldsymbol{R},|s| \rightarrow \infty$. Hence $N_{m}^{k+1} x=0$ for each $x \in D_{m}$, completing the proof of Lemma 2.9.

Lemma 2.10. For all $m \geqq k+2,|x|_{m, T}=|x|_{k+2, T}$, hence $D=D_{m}=D_{k+2}$.

Proof. Fix $m \geqq k+2$. By Lemma 2.2(c) and Lemma 2.9,

$$
\begin{aligned}
T(f) x & =\sum_{j=0}^{k} \frac{1}{j !} \int_{\sigma(T)} f^{(j)}(t) d E_{m}(t) N_{m}^{j} x \\
& =\sum_{j=0}^{k} \frac{1}{j !} S_{m}\left(f^{(j)}\right) N_{m}^{j} x
\end{aligned}
$$

for all $f \in C^{m}$ and $x \in D_{m}$.

By (2.7) and Lemma 2.9(ii),

$$
\left|\int_{\sigma(T)} f^{(j)}(t) d E_{m}(t) N_{m}^{j} x\right|_{m, T} \leqq \sup _{\sigma(T)}\left|f^{(j)}\right||x|_{m, T},
$$

and therefore

$$
|T(f) x|_{m, T} \leqq|f|_{k, T}|x|_{m, T} \text { for all } f \in C^{m}, x \in D_{m} .
$$

Hence

$$
\begin{aligned}
|x|_{k+2, T} & =\sup \left\{|T(f) x| ; f \in C^{k+2},|f|_{k+2, T} \leqq 1\right\} \\
& =\sup \left\{|T(f) x| ; f \in C^{m},|f|_{k+2, T} \leqq 1\right\} \\
& \leqq|x|_{m, T}
\end{aligned}
$$

for all $x \in D_{m}$. Hence $|x|_{m, T}=|x|_{k+2, T}$ for all $x \in D_{m}$. Q.E.D.

Definition 2.4. We let $S=S_{k+2}, N=N_{k+2}, E=E_{k+2}, S(f)=S_{k+2}(f)$ for $f \in B(\sigma(T))$ and $\|x\|=|x|_{k+2, T}$ for $x \in D$.

By Lemma 2.10, $(D,\|x\|)$ is a normed linear space. Also $S, N, E(\delta)$ and $S(f)$ are in $T(D)$ for all $\delta \in \mathscr{B}$ and $f \in B(\sigma(T))$. By Lemma 2.4, we have $\|S x\| \leqq|T|_{\mathrm{sp}}\|x\|$, $\|N x\| \leqq\|x\|,\|S(f) x\| \leqq \sup _{\sigma(T)}|f| \cdot\|x\|$ and $\|E(\delta) x\| \leqq\|x\|$ for all $x \in D$, $f \in \boldsymbol{B}(\sigma(T))$ and $\delta \in \mathscr{B}$.

By the remark following Corollary 2.6, we have a decomposition $T \mid D=S+N$, which satisfies (2.2) and (2.3) by Lemma 2.9, and (2.4) by Lemma 2.8 and Corollary 2.6 .

LEMma 2.11. For each $x \in D$, the map $f \rightarrow T(f) x$ of $C^{k+2}$ into $X$ has an extension as a continuous linear map of $C^{k}$ into $D$, which is also continuous as a map into $(D,\|\cdot\|)$. The extended map is given by $(2.5)$, and $f \rightarrow T(f)$ is an algebra homomorphism of $C^{k}$ into $T(D)$. 
Proof. By Lemma 2.10, Equation (2.13) and Definition 2.4, the right-hand side of (2.5) gives the required extension. Its continuity properties follow from Lemma 2.4 .

We consider now the map $f \rightarrow T(f)$ of $C^{k}$ into $T(D)$ given by (2.5), i.e.,

$$
T(f)=\sum_{j=0}^{k} \frac{1}{j !} S\left(f^{(j)}\right) N^{j}, \quad f \in C^{k} .
$$

This map is clearly well defined and linear. Its multiplicativity follows from Lemmas 2.8 and 2.9: for $f, g \in C^{k}$,

$$
\begin{aligned}
T(f g) & =\sum_{j=0}^{k} S\left\{\sum_{h=0}^{j} \frac{f^{(h)} g^{(j-h)}}{h !(j-h) !}\right\} N^{j} \\
& =\sum_{j=0}^{k} \sum_{h=0}^{j} \frac{1}{h !(j-h) !} S\left(f^{(h)}\right) S\left(g^{(j-h)}\right) N^{j} \\
& =\sum_{h=0}^{k} \frac{1}{h !} S\left(f^{(h)}\right) \sum_{p=0}^{k-h} \frac{1}{p !} S\left(g^{(p)}\right) N^{p+h} .
\end{aligned}
$$

Since $N^{k+1}=0$, the inner summation may be extended up to $k$. Thus

$$
T(f g)=\sum_{h=0}^{k} \frac{1}{h !} S\left(f^{(h)}\right) T(g) N^{h} .
$$

Since $N$ commutes with $T(g)$ by Lemma 2.9(i), we obtain

$$
T(f g)=\sum_{h=0}^{k} \frac{1}{h !} S\left(f^{(h)}\right) N^{h} T(g)=T(f) T(g), \quad \text { Q.E.D. }
$$

Proof of Theorem 2.1, completed. It remains to prove the "maximalityuniqueness" assertion. Let $W$ be an invariant linear manifold for $T$, and let $S_{1}, N_{1}$ and $E_{1}$ be related to $T$ and $W$ as stated for $S, N, E$ and $D$ in (2.1)-(2.4). It follows from (2.1) and (2.2) for $S_{1}$ and $N_{1}$ that

$$
T^{n} x=\sum_{j=0}^{n}\left(\begin{array}{l}
n \\
j
\end{array}\right) S_{1}^{n-j} N_{1}^{j} x
$$

for all $x \in W$ and $n=0,1,2 \cdots\left(N_{1}^{0}=S_{1}^{0}=I\right.$ by definition).

Let $q$ be an integer $\geqq k$. Then for $x \in W$,

$$
\begin{aligned}
\sum_{n=0}^{q} \frac{(i t)^{n}}{n !} T^{n} x & =\sum_{n=0}^{q} \frac{(i t)^{n}}{n !} \sum_{j=0}^{n}\left(\begin{array}{c}
n \\
j
\end{array}\right) S_{1}^{n-j} N_{1}^{j} x \\
& =\sum_{j=0}^{k}\left(\sum_{r=0}^{q-j} \frac{(i t)^{r} S_{1}^{r}}{r !}\right) \frac{(i t)^{j} N_{1}^{j} x}{j !} \\
& =\sum_{j=0}^{k}\left\{\int_{\sigma(T)} \sum_{r=0}^{q-j} \frac{(i t s)^{r}}{r !} d E_{1}(s)\right\} \frac{(i t)^{j} N_{1}^{j} x}{j !}
\end{aligned}
$$

where we used (2.3) and (2.4) for $S_{1}$ and $N_{1}$. 
Fix $j, 0 \leqq j \leqq k$. Since $\sigma(T)$ is compact, the dominated convergence theorem for vector measures $[3, \mathrm{p} .328]$ gives:

$$
\lim _{q \rightarrow \infty} \int_{\sigma(T) r=0} \sum_{r=0}^{j} \frac{(i t s)^{r}}{r !} d E_{1}(s) N_{1}^{j} x=\int_{\sigma(T)} e^{i t s} d E_{1}(s) N_{1}^{j} x
$$

(in the strong topology of $X$ ) for each fixed $x \in W$ and $t \in R$.

Hence, taking $q \rightarrow \infty$ in (2.15), we obtain

for all $t \in R$ and $x \in W$.

$$
e^{i t T} x=\sum_{j=0}^{k} \frac{(i t)^{j}}{j !} \int_{\sigma(T)} e^{i t s} d E_{1}(s) N_{1}^{j} x
$$

For $f \in C_{0}^{k+2}$ and $x \in W$, we obtain (cf. (1.5)):

i.e.,

$$
\begin{aligned}
T(f) x & =\int_{R} \hat{f}(t) e^{i t T} d t \\
& =\sum_{j=0}^{k} \frac{1}{j !} \int_{R} \hat{f}(t)(i t)^{j} \int_{\sigma(T)} e^{i t s} d E_{1}(s) N_{1}^{j} x d t \\
& =\sum_{j=0}^{k} \frac{1}{j !} \int_{\sigma(T)}\left\{\int_{R} \hat{f}(t)(i t)^{j} e^{i t s} d t\right\} d E_{1}(s) N_{1}^{j} x,
\end{aligned}
$$

$$
T(f) x=\sum_{j=0}^{k} \frac{1}{j !} \int_{\sigma(T)} f^{(j)}(s) d E_{1}(s) N_{1}^{j} x \text { for all } f \in C_{0}^{k+2}
$$

and $x \in W$. The interchange of the order of integration and the use of the Fourier inversion theorem are justified since $f \in C_{0}^{k+2}$ and $E_{1}(\cdot) N_{1}^{j} x$ is a bounded measure with compact support $\sigma(T)$, for $x \in W$ and $0 \leqq j \leqq k$ fixed.

For $f \in C^{k+2}, T(f)$ is defined as $T\left(f_{0}\right)$ for any $f_{0} \in C_{0}^{k+2}$ which coincides with $f$ in some open interval containing $\sigma(T)=\Sigma$ (cf. proof of Lemma 2.11 in [7]). Therefore

$$
T(f) x=\sum_{j=0}^{k} \frac{1}{j !} \int_{\sigma(T)} f^{(j)}(s) d E_{1}(s) N_{1}^{j} x
$$

for all $f \in C^{k+2}$ and $x \in W$.

For $x \in W$ fixed, let $M(x)>0$ be a common bound for the vector measures $E_{1}(\cdot) N_{1}^{j} x, 0 \leqq j \leqq k$. Then, by $(2.16)$,

$$
|T(f) x| \leqq 4 M(x)|f|_{k, T} \leqq 4 M(x)|f|_{k+2, T}
$$

for all $f \in C^{k+2}$. Hence $|x|_{k+2, T} \leqq 4 M(x)$, and $x \in D_{k+2}=D$. This proves that $W \subset D$.

By (2.5), (2.16) and Lemma 3.7 in [7],

$$
E_{1}(\delta) N_{1}^{j} x=E(\delta) N^{j} x
$$

for all $\delta \in \mathscr{B}, 0 \leqq j \leqq k$ and $x \in W$ (since $\sigma(T)$ is a Lebesgue null set). 
In particular $(j=0), E_{1}(\delta) x=E(\delta) x$ for all $\delta \in \mathscr{B}$ and $x \in W$; hence $S_{1} x=S x$ by (2.4) and $N_{1} x=N x$ by (2.1) for all $x \in W \subset D$. This completes the proof of Theorem 2.1 .

The maximality-uniqueness assertion of Theorem 2.1 justifies the following Definition 2.5. We call $S, N$ and $E$ respectively the scalar part, the nilpotent part and the resolution of the identity of $T$ on the Jordan manifold $D$.

Keeping in mind the usual definition of a resolution of the identity (cf. [1], [2]), the following proposition is of some interest.

Proposition 2.12. If the nilpotent part (or the scalar part) of $T$ is closable, then $E(\delta)$ commutes with $S, N$ and $S(f)$ for all $\delta \in \mathscr{B}$ and $f \in C(\sigma(T))$, and moreover

for all $\delta, \varepsilon \in \mathscr{B}$.

$$
E(\delta \cap \varepsilon)=E(\delta) E(\varepsilon)
$$

Proof. Fix $\delta \in \mathscr{B}$, and let $c_{\delta}$ denote its characteristic function. Choose $f_{n} \in C$ such that $\left|f_{n}\right| \leqq 1$ and $f_{n} \rightarrow c_{\delta}$ pointwise $\left({ }^{2}\right)$ on $\sigma(T)$. By the dominated convergence theorem for vector measures, $S\left(f_{n} g\right) x \rightarrow S\left(c_{\delta} g\right) x$ strongly, for all $x \in D$ and $g \in C(\sigma(T))$. Since $S(g) D \subset D$, it follows from Lemma 2.8 that

$$
S\left(f_{n} g\right) x=S\left(f_{n}\right) S(g) x \rightarrow E(\delta) S(g) x .
$$

Hence, for all $g \in C(\sigma(T))$,

$$
S\left(c_{\delta} g\right)=E(\delta) S(g) .
$$

Fix $x \in D$, and let $y_{n}=S\left(f_{n}\right) x-E(\delta) x$. Then $y_{n} \in D$ and $y_{n} \rightarrow 0$ (take $g \equiv 1$ above). Also

$$
S y_{n}=S\left(t f_{n}(t)\right) x-S E(\delta) x \rightarrow E(\delta) S x-S E(\delta) x
$$

(take $g(t) \equiv t$ above). But $S$ is closable (either by hypothesis, or because $S=T \mid D-N$ where $T \mid D$ is bounded and $N$ is closable). Therefore $E(\delta) S x=S E(\delta) x$ for each $x \in D$. Thus $E(\delta)$ commutes with $S$, and since it commutes with $T$, it commutes also with $N=T \mid D-S$.

Given $\varepsilon>0$ and $f \in C(\sigma(T))$, choose a polynomial $p$ such that $\sup _{\sigma(T)}|f-p|<\varepsilon$. Since $E(\delta)$ commutes with $p(S)$, we obtain for each $x \in D$ :

$$
|E(\delta) S(f) x-S(f) E(\delta) x| \leqq|E(\delta) S(f-p) x|+|S(f-p) E(\delta) x| \leqq 2 \varepsilon\|x\|
$$

by the remarks following Definition 2.4.

Hence $E(\delta)$ commutes with $S(f)$ for all $f \in C(\sigma(T))$. Therefore, by (2.17), $S\left(c_{\delta} g\right) x=S(g) E(\delta) x$, i.e.,

$$
\int_{\sigma(T)} g(t) c_{\delta}(t) d E(t) x=\int_{\sigma(T)} g(t) d E(t) E(\delta) x
$$

for all $g \in C(\sigma(T))$ and $x \in D$.

(2) Exceptional sets are routinely disposed of. Details are left to the reader. 
Fixing $x \in D$, it follows from the uniqueness of the Riesz representation that for all $\varepsilon \in \mathscr{B}$

$$
\int_{\varepsilon} c_{\delta}(t) d E(t) x=\int_{\varepsilon} d E(t) E(\delta) x
$$

i.e., $E(\varepsilon \cap \delta) x=E(\varepsilon) E(\delta) x$ for all $\varepsilon, \delta \in \mathscr{B}$ and $x \in D$, Q.E.D.

Since $N=0$ is trivially closable, we have

Corollary 2.13. Suppose $T$ satisfies Condition (1.1) for $k=0$ (i.e., $\left.\left|e^{i t T}\right|=O(1), t \in R\right)$. Then $E(\delta)$ commutes with $S(f)$ for all $f \in C(\sigma(T))$, and $E(\delta \cap \varepsilon)=E(\delta) E(\varepsilon)$ for all $\delta, \varepsilon \in \mathscr{B}$.

Theorem 2.1 may be given a version fitting into Dunford's theory of spectral operators [1], [2].

Definition 2.6. The Jordan space for $T$ is the completion $Y$ of the normed linear space $(D,\|\cdot\|)$.

If $V \in B(X)$ commutes with $T$, we know that $V D \subset D$ and that $V$ is continuous on $(D,\|\cdot\|)$. Let $V_{Y}$ be the unique extension of $V \mid D$ as a bounded linear operator on $Y$. If $\left\|V_{Y}\right\|$ denotes the norm of $V_{Y}$ as an element of $B(Y)$, then $\left\|V_{Y}\right\| \leqq|V|$. Similarly, $S, N, E(\delta)$ and $S(f)$ are bounded on $(D,\|\cdot\|)$; denote their unique continuous extension to $Y$ by the corresponding letter with a subscript $Y$. By the remark following Definition 2.4 , we have $\left\|S_{Y}\right\| \leqq|T|_{\text {sp }},\left\|N_{Y}\right\| \leqq 1$, $\left\|E(\delta)_{Y}\right\| \leqq 1$ and $\left\|S(f)_{Y}\right\| \leqq \sup _{\sigma(T)}|f|^{\prime}(\delta \in \mathscr{B}, f \in B(\sigma(T)))$. By (2.1)-(2.3), $T_{Y}=S_{Y}+N_{Y}, S_{Y}$ commutes with $N_{Y}$, and $N_{Y}^{k+1}=0$. However, we get into trouble when we try to show that $E(\cdot)_{Y}$ is a resolution of the identity in the sense of $\left[1\right.$, pp. 324-325]. First, we do not know whether $E(\delta \cap \varepsilon)_{Y}=E(\delta)_{Y} E(\varepsilon)_{Y}$ in general. A more serious difficulty is the countable additivity of $y^{*} E(\cdot)_{Y} y$ for $y^{*}$ in a total linear manifold $\Gamma \subset Y^{*}$. A natural choice of $\Gamma$ is as follows: each $x^{*} \in X^{*}$ induces a unique $x_{Y}^{*} \in Y^{*}$ with norm $\left\|x_{Y}^{*}\right\| \leqq\left|x^{*}\right|$ (since $|x| \leqq\|x\|$ for $x \in D)$. Let $\Gamma=\left\{x_{Y}^{*} \mid x^{*} \in X^{*}\right\}$. It is easily seen that $x_{Y}^{*} E(\cdot)_{Y} y$ is countably additive on $\mathscr{B}$, for each $y \in Y$ and $x_{Y}^{*} \in \Gamma$, and that $x_{Y}^{*} S(f)_{Y} y=\int_{\sigma(T)} f(t) d x_{Y}^{*} E(t)_{Y} y$ for all $f \in B(\sigma(T)), y \in Y$ and $x_{Y}^{*} \in \Gamma$. However $\Gamma$ may not be total in $Y^{*}$. Indeed, let $X$ and $T$ be chosen such that the norms $|\cdot|$ and $\|\cdot\|$ are not equivalent (cf. §3). We may then select $x_{n} \in D$ such that $x_{n} \rightarrow 0$ in $X$ and $x_{n} \rightarrow y \neq 0$ in $Y$. For each $x^{*} \in X^{*}, x^{*} x_{n} \rightarrow 0$, hence $x_{Y}^{*} y=\lim x^{*} x_{n}=0$ for all $x_{Y}^{*} \in \Gamma$, but $y \neq 0$, i.e., $\Gamma$ is not total in $Y^{*}$.

However, a slight modification of the proof of Theorem 2.1 yields to the following

THEOREM 2.14. $T_{Y}^{*}$ is spectral of class $Y$ and type $k$ (in this theorem, $X$ does not need to be reflexive).

Outline of proof. Let us simplify first the notations by omitting the subscript $Y$. Fix $m=k+2$ and let $\boldsymbol{A}_{m}$ be as in the proof of Theorem 2.1. For $y \in Y$ and $y^{*} \in Y^{*}$ fixed, consider the map 


$$
\pi\left(y, y^{*}\right): f \in A_{m} \rightarrow y^{*} T(f) y
$$

where $f_{j}=f^{(j)} \mid \sigma(T)$. By (1.2), this map is well defined, linear and continuous. We conclude as in the proof of Lemma 2.2 that there exist $m+1$ regular finite Borel measures $\mu_{j}=\mu_{j}\left(\cdot \mid y, y^{*}\right)(j=0, \cdots, m)$ on $\boldsymbol{R}$, which are supported by $\sigma(T)$ and satisfy

and

$$
\operatorname{var} \mu_{j}\left(\cdot \mid y, y^{*}\right) \leqq\|y\|\left\|y^{*}\right\|,
$$

$$
y^{*} T(f) y=\sum_{j=0}^{m} \frac{1}{j !} \int_{\sigma(T)} f^{(j)} d \mu_{j}, \quad f \in C^{m} .
$$

Moreover, this representation is unique, and therefore $\mu_{j}\left(\delta \mid y, y^{*}\right)$ is a continuous bilinear form on $Y \times Y^{*}$ for each $\delta \in \mathscr{B}$ and $j=0, \cdots, m$. Let $F_{j}(\delta)$ be the unique bounded linear operator on $Y^{*}$ such that $\mu_{j}\left(\delta \mid y, y^{*}\right)=\left\langle F_{j}(\delta) y^{*}, y\right\rangle$ for all $y$ and $y^{*}$ (the pairing of $Y$ and $Y^{*}$ is denoted by $\langle\cdot, \cdot\rangle$ ). The $F_{j}(\cdot)$ are uniformly bounded additive set functions with the property that $\left\langle F_{j}(\cdot) y^{*}, y\right\rangle$ are regular (countably additive) Borel measures on $\boldsymbol{R}$ supported by $\sigma(T)$ (for all $j=0, \cdots, m ; y \in Y$ and $\left.y^{*} \in Y^{*}\right)$. Moreover, for all $f \in C^{m}$,

$$
T(f)^{*}=\sum_{j=0}^{m} \frac{1}{j !} \int_{\sigma(T)} f^{(j)} d F_{j},
$$

where, for $h \in C(\sigma(T)), \int_{\sigma(T)} h d F_{j}$ is the unique element of $B\left(Y^{*}\right)$ defined by

$$
\left\langle\int_{\sigma(T)} h d F_{j} y^{*}, y\right\rangle=\int_{\sigma(T)} h d\left\langle F_{j} y^{*}, y\right\rangle
$$

for all $y$ and $y^{*}$. In particular, for all $s \varepsilon R$,

where

$$
\left(e^{i s T}\right)^{*}=\sum_{j=0}^{m} \frac{(i s)^{j}}{j !} F_{j}(s)
$$

$$
F_{\hat{j}}(s)=\int_{\sigma(T)} e^{i s t} d F_{j}(t) .
$$

Trivial changes in the proof of Lemma 2.7 yield to the identities

$$
\begin{aligned}
F_{j}(s) F_{\hat{h}}(u) & =F_{j+\hat{h}}(s+u) & & \text { for } j+h \leqq m, \\
& =0 & & \text { for } j+h>m,
\end{aligned}
$$

for all $j, h=0, \cdots, m$ and $s, u \in R$.

It follows as in the proof of Lemma 2.8 that the $\operatorname{map} f \rightarrow S(f)=\int_{\sigma(T)} f d F_{0}$ of $C(\sigma(T))$ into $B\left(Y^{*}\right)$ is multiplicative; since $F_{0}(\cdot)$ is uniformly bounded, the extension of this map to $B(\sigma(T))$ is also multiplicative, and therefore

$$
F_{0}^{\prime}(\delta \cap \varepsilon)=S\left(c_{\delta \cap \varepsilon}\right)=S\left(c_{\delta} c_{\varepsilon}\right)=S\left(c_{\delta}\right) S\left(c_{\varepsilon}\right)=F_{0}(\delta) F_{0}(\varepsilon)
$$


for all $\delta, \varepsilon \in \mathscr{B}$. Taking $s=0$ in (2.18), we see that $F_{0}(\sigma(T))=I$ (the identity of $B\left(Y^{*}\right)$ ). Thus $F=F_{0}$ is a spectral measure on $R$.

Taking $f(t) \equiv t$ in $\left(^{*}\right)$, we obtain

$$
T^{*}=\int_{\sigma(T)} t d F(t)+F_{1}(\sigma(T))=S+N .
$$

It follows from (2.19), much as in the proof of Lemma 2.9, that $F_{j}=N^{j} F=F N^{j}$ $(j=0, \cdots, m)$. Hence $S$ commutes with $N$, and since $e^{i s S}=\int_{\sigma(T)} e^{i s t} d F(t)$, we obtain from (2.18):

$$
\left(e^{i s T}\right)^{*} e^{-i s S}=\sum_{j-0}^{k+2} \frac{(i s)^{j}}{j !} N^{j} .
$$

But $\left\|\left(e^{i s T}\right)^{*} e^{-i s S}\right\| \leqq\left\|\left(e^{i s T}\right)^{*}\right\|=\left\|e^{i s T}\right\| \leqq\left|e^{i s T}\right|=O\left(|s|^{k}\right)$. Therefore $N^{k+1}=0$, and the proof is complete, by Theorem 8 in [1].

The (perhaps) unusual method we used to prove Theorem 2.1 was needed essentially in order to handle nonisolated points of the spectrum. In case there are isolated points in $\sigma(T)$, a result of the same nature (without the maximalityuniqueness assertion) may be obtained by a more classical method, without requiring the reflexivity of $X$.

Notation. Let $\sigma_{0}(T)$ denote the set of isolated points in $\sigma(T)$. If $\sigma_{0}(T) \neq \varnothing$, let $Z_{0}$ denote the linear span of $E(\lambda) X$ for $\lambda$ running in $\sigma_{0}(T)$; if $\sigma_{0}(T)=\varnothing$, we let $Z_{0}=\{0\}$.

Suppose $T$ satisfies Condition (1.1).

It follows from the proof of Theorem 1.5 that if every nonzero point of $\sigma(T)$ is isolated, then the closure of $Z_{0}$ contains the closed range of $T^{k+1}$. In case $k=0$ and $X$ is reflexive, $Z_{0}+\mathscr{N}$ is dense in $X$. An alternative description of $Z_{0}$ is given in the following

Proposition 2.15. Let $T \in B(X)$ satisfy (1.1). Then

(a) each isolated point of $\sigma(T)$ is a pole of order $\leqq k+1$ of the resolvent; and

(b) $Z_{0}$ is the linear span of the root vectors corresponding to the points of $\sigma_{0}(T)$.

Proof. Let $\lambda \in \sigma_{0}(T)$. Since $\lambda$ is real, $\lambda I-T$ satisfies (1.1), hence (1.4) (by Lemma 1.9). Since $\sigma(T \mid E(\lambda) X)=\{\lambda\}$, the operator $(\lambda I-T) \mid E(\lambda) X$ is quasinilpotent. Hence, by Lemma 1.8 ,

$$
(\lambda I-T)^{k+1} E(\lambda)=0 .
$$

By Theorem 18, p. 573 in [3], this implies that $\lambda$ is a pole of order $\leqq k+1$ of $R(\lambda ; T)$. Since, in this case, the range of $E(\lambda)$ coincides with the root space associated with $\lambda$, the proposition follows. 
Proposition 2.16. Let $T \in B(X)$ satisfy (1.1). Then all the conclusions of Theorem 2.1 (except for the maximality-uniqueness assertion) are valid with $Z_{0}$ replacing $D$.

Proof. For $x \in Z_{0}$, let

$$
\sigma(x)=\left\{\lambda \in \sigma_{0}(T) \mid E(\lambda) x \neq 0\right\} .
$$

Clearly, $\sigma(x)$ is either void or finite, and

$$
x=\sum_{\lambda \in \sigma(x)} E(\lambda) x, \quad x \in Z_{0} .
$$

Let $S, N \in T\left(Z_{0}\right)$ be defined by

$$
\begin{aligned}
S x & =\sum_{\lambda \in \sigma(x)} \lambda E(\lambda) x, \quad x \in Z_{0}, \\
N & =T \mid Z_{0}-S .
\end{aligned}
$$

Then (2.1) and (2.2) are obvious. We prove next by induction that for all $x \in Z_{0}$ and $j=0,1,2, \cdots$,

$$
N^{j} x=\sum_{\lambda \in \sigma(x)}(T-\lambda I)^{j} E(\lambda) x .
$$

For $j=0$, this reduces to (2.21). Assuming (2.23) true for some $j$, we obtain (for $x \in Z_{0}$ ):

$$
\begin{aligned}
N^{j+1} x & =N \sum_{\lambda \in \sigma(x)}(T-\lambda I)^{j} E(\lambda) x=\sum_{\lambda \in \sigma(x)}(T-\lambda I)^{j} E(\lambda) N x \\
& =\sum_{\lambda \in \sigma(x)}(T-\lambda I)^{j} E(\lambda) \sum_{\mu \in \sigma(x)}(T-\mu I) E(\mu) x \\
& =\sum_{\lambda \in \sigma(x)}(T-\lambda I)^{j+1} E(\lambda) x .
\end{aligned}
$$

Now, by (2.23) and (2.20), $N^{k+1}=0$.

For $\delta \in \mathscr{B}$, define

$$
E(\delta) x=\sum_{\lambda \in \sigma(x) \cap \delta} E(\lambda) x, \quad x \in Z_{0} .
$$

Then $E$ is a generalized spectral measure as in Theorem 2.1 (with the additional property $E(\delta \cap \varepsilon) x=E(\delta) E(\varepsilon) x$ for all $\delta, \varepsilon \in \mathscr{B}$ and $\left.x \in Z_{0}\right)$, and $S$ satisfies (2.4). Since (2.5) follows from (2.1)-(2.4) (see proof of "maximality" in Theorem 2.1 , the proof is complete.

REMARKS. 1. By the uniqueness assertion in Theorem 2.1, the transformations $S, N$ and $E(\delta)$ obtained there are extensions of the respective transformations obtained here (if the hypotheses of Theorem 2.1 are satisfied).

2. If $\sigma_{0}(T)=\sigma(T)$ (i.e., if $\sigma(T)$ is a finite set), then $S, N$ and $E(\delta)$ are in $B(X)$, and $T$ is spectral of type $k$. 
The case $k=0$ has a distinguished position if $X$ is a Hilbert space. By Theorem 5 in [6], the condition $\left|e^{i t T}\right|=O(1)(t \in R)$ by itself is then sufficient for $T$ to be spectral of type $k=0$ (i.e., similar to a hermitian operator). This is no longer true (in Hilbert space) for $k \geqq 1$ (cf. §3). In Banach space, whether reflexive or not, this breaks down even for $k=0$ (cf. [6, p. 176]).

Let $\boldsymbol{R}[t]$ denote the ring of polynomials over $\boldsymbol{R}$. We may rewrite Condition (1.1) for $k=0$ in the form $\left|e^{i p(T)}\right| \leqq M<\infty$ for all $p \in R[t]$ of degree $\leqq 1$. If we require this latter condition to hold for all $p \in \boldsymbol{R}[t]$, we obtain a criterion for spectrality which is valid in any weakly complete Banach space.

THEOREM 2.17. $T \in B(X)$ is of class $C$ and has real spectrum if and only if

$$
\sup _{p \in \mathbb{R}[t]}\left|e^{i p(T)}\right|<\infty
$$

If $X$ is weakly complete, Condition $\left(^{*}\right)$ is necessary and sufficient for $T$ to be spectral of scalar type with real spectrum.

Proof. If $X$ is weakly complete, $T$ is of class $C$ if and only if it is spectral of scalar type (cf. Theorem 3.2 in [7]). Thus, only the first statement of the theorem needs proof.

If $T$ is of class $C$, it is of class $C(\sigma(T)$ ) (cf. Lemma 2.7 and Definition 1.2 in [7]). Let $f \rightarrow T(f)$ be its $C(\sigma(T))$-operational calculus, and let $M$ be the latter's norm. Then for all $p \in \boldsymbol{R}[t]$,

$$
\left|e^{i p(T)}\right|=\left|T\left(e^{i p(t)}\right)\right| \leqq M \sup _{t \in \sigma(T)}\left|e^{i p(t)}\right|=M
$$

if $\sigma(T)$ is real.

Conversely, suppose $T$ satisfies (*). Let

$$
M=\sup _{p \in \mathbb{R}[t]}\left|e^{i p(T)}\right| .
$$

In particular, we have $\left|e^{i t T}\right| \leqq M$ for all $t \in R$, hence $T$ has real spectrum.

Let $A_{r}(T)$ denote the uniformly closed real subalgebra of $B(X)$ generated by $I$ and $T$. Suppose $V \in A_{r}(T)$ is quasi-nilpotent. Choose $p_{n} \in R[t]$ such that $p_{n}(T) \rightarrow V$ in $B(X)$. Then $\left|e^{i t V}\right|=\lim _{n}\left|e^{i t p} n^{(T)}\right| \leqq M$ for all $t \in R$. By Lemmas 1.9 and 1.8, we must have $V=0$. Thus $A_{r}(T)$ is semisimple. It follows that its complexification $A(T)$ is semisimple.

Next, for an arbitrary element $V$ of $A_{r}(T)$, we have $\left|e^{i t V}\right| \leqq M$ for all $t \in R$; therefore the integral

$$
R(V)=\frac{1+i}{2}(2 \pi)^{-1 / 2} \int_{0}^{\infty} \frac{e^{i t V}-1}{t^{3 / 2}} d t
$$


converges absolutely in $B(X)$, and defines an element of $A(T)$. By Theorem 2 in [9], we have

$$
R(V)^{2}=V
$$

For $V \neq 0$, we have

$$
|R(V)| \leqq \frac{1}{2}(\pi)^{-1 / 2}\left\{\int_{0}^{|V|^{-1}} \frac{\left|e^{i t V}-1\right|}{t^{3 / 2}} d t+\int_{|V|^{-1}}^{\infty} \frac{\left|e^{i t V}\right|+1}{t^{3 / 2}} d t\right\} .
$$

For $0 \leqq t \leqq|V|^{-1}$,

$$
\begin{aligned}
\left|e^{i t V}-1\right| & \leqq t|V|\left\{1+\frac{t|V|}{2 !}+\frac{(t|V|)^{2}}{3 !}+\cdots\right\} \\
& \leqq t|V|\left\{1+\frac{1}{2 !}+\frac{1}{3 !}+\cdots\right\} \\
& \leqq(e-1)|V| t
\end{aligned}
$$

and therefore the first integral above is not larger than $2(e-1)|V|^{1 / 2}$. The second integral is $\leqq 2(M+1)|V|^{1 / 2}$. Hence

$$
|R(V)| \leqq K|V|^{1 / 2}
$$

for all $V \in A_{r}(T)$, where $K=(M+e) \pi^{-1 / 2}$.

By (2.25), $R\left(V^{2}\right)^{2}=V^{2}$. Since $R\left(V^{2}\right) \in A(T)$ for $V \in A_{r}(T)$ and since $A(T)$ is semisimple, it follows that $R\left(V^{2}\right)= \pm V$. Therefore, applying (2.26) with $V^{2}$ instead of $V$, we obtain $|V|^{2} \leqq K^{2}\left|V^{2}\right|$, and thus $|V| \leqq K^{2}|V|_{\text {sp }}$ for all $V \in A_{r}(T)$. It follows that $A(T)$ is isometrically isomorphic to $C(\mathscr{M})$, where $\mathscr{M}$ is the maximal ideal space of $A(T)$; hence $T$ is of class $C$. Alternatively, taking $V=p(T)$, we get

$$
|p(T)| \leqq K^{2}|p(T)|_{\mathrm{sp}}=K^{2} \sup _{\sigma(T)}|p(t)|
$$

for all $p \in R[t]$. Therefore the map $p \rightarrow p(T)$ extends as a continuous representation $f \rightarrow f(T)$ of $C(\sigma(T))$ into $B(X)$, with norm $\leqq K^{2}$. Q.E.D.

We conclude this section with another characterization of operators of class $C$ with real spectrum. Without loss of generality, we may consider the case where $\sigma(T) \subset[0,1]$.

THEOREM 2.18. Let $T \in B(X)$ be such that $\sigma(T) \subset[0,1]$. Then $T$ is of class $C$ (i.e., $T$ is spectral of scalar type for $X$ weakly complete) if and only if there is an $M>0$ such that

$$
\sum_{j=0}^{n}\left(\begin{array}{l}
n \\
j
\end{array}\right)\left|x^{*} T^{J}(I-T)^{n-j} x\right| \leqq M
$$

for all unit vectors $x \in X$ and $x^{*} \in X^{*}$, and all $n=0,1,2, \cdots$. 
Proof. Let $\Delta$ denote the difference operator acting on complex sequences: if $\mu=\left\{\mu_{n}\right\}, \Delta \mu$ is the sequence with $n$th component $(\Delta \mu)_{n}=\mu_{n}-\mu_{n+1}, n=0,1,2, \cdots$. The iterates of $\Delta$ are denoted by $\Delta^{k}(k=1,2, \cdots)$, and $\Delta^{0} \mu=\mu$ for all $\mu$.

Fix unit vectors $x \in X$ and $x^{*} \in X^{*}$. Let $\mu$ be the sequence defined by

$$
\mu_{n}=x^{*} T^{n} x, \quad n=0,1,2, \cdots
$$

Then

$$
\left(\Delta^{n-j} \mu\right)_{j}=x^{*} T^{j}(I-T)^{n-j} x
$$

for $j=0, \cdots, n$ and $n=0,1,2, \cdots$.

Therefore, $(* *)$ is equivalent to the condition

$$
\sum_{j=0}^{n}\left(\begin{array}{l}
n \\
j
\end{array}\right)\left|\left(\Delta^{n-j} \mu\right)_{j}\right| \leqq M, \quad n=0,1,2, \cdots .
$$

This is Hausdorff's classical criterion for $\mu$ to be a moment sequence on $[0,1]$. Thus, Condition $\left({ }^{* *}\right)$ is equivalent to the existence of a (unique) finite regular Borel measure $\alpha=\alpha\left(\cdot \mid x, x^{*}\right)$ such that $\operatorname{var} \alpha \leqq M$ and

$$
x^{*} T^{n} x=\int_{0}^{1} t^{n} d \alpha\left(t \mid x, x^{*}\right), \quad n=0,1,2, \cdots,
$$

(for all unit vectors $x$ and $x^{*}$ ).

If $T$ is of class $C,(* * *)$ is a direct consequence of the Riesz representation theorem. Conversely, if $T$ satisfies $\left({ }^{* *}\right)$, then $x^{*} p(T) x=\int_{0}^{1} p(t) d \alpha\left(t \mid x, x^{*}\right)$ for all $p \in R[t]$ and all unit vectors $x$ and $x^{*}$. Hence $|p(T)| \leqq M \sup _{[0,1]}|p(T)|$ for all $p \in R[t]$, which implies that $T$ is of class $C$ (cf. end of previous proof).

\section{Examples.}

3.1. We remarked above that in Hilbert space, Condition (1.1) for $k=0$ is sufficient by itself for the spectrality of $T$. The following example, which is due C. A. McCarthy, shows that this is no longer true for $k=1$, even when $\sigma(T)$ is a sequence with 0 as its only limit point and when $X$ is a separable Hilbert space. Thus $D$ does not generally coincide with $X$, even in this restricted situation (in this case however, $D$ is dense in $X$ ).

Let $X=\sum_{n=1}^{\infty} \oplus H_{n}$, where $H_{n}$ is a copy of the 2-dimensional Hilbert space $C^{2}$. Consider on $H_{n}$ the projections $E_{n}$ and $E_{-n}$ defined by the matrices

$$
\left(\begin{array}{ll}
1 & n \\
0 & 0
\end{array}\right) \text { and }\left(\begin{array}{cc}
0 & -n \\
0 & 1
\end{array}\right)
$$

respectively $(n=1,2, \cdots)$. Clearly,

$$
E_{-n} E_{n}=E_{n} E_{-n}=0, \quad E_{ \pm n}^{2}=E_{ \pm n} \text { and } E_{n}+E_{-n}=I .
$$

Let $T_{n}=\left(E_{n}-E_{-n}\right) / n$ and $T=\sum_{n=1}^{\infty} \oplus T_{n}$. Then $\sigma(T)=\{0, \pm 1 / n ; n=1,2, \cdots\}$ 
and $T$ is of class $C^{\prime}$ : its $C^{\prime}$-operational calculus is given by $T(f)=\sum_{n=1}^{\infty} \oplus T(f)_{n}$, where

$$
T(f)_{n}=f\left(\frac{1}{n}\right) E_{n}+f\left(-\frac{1}{n}\right) E_{-n}
$$

$\left(f \in C^{\prime}, n=1,2, \cdots\right)$. The continuity of the map $f \rightarrow T(f)$ follows from the mean value theorem, which gives the estimate

$$
|T(f)| \leqq \max _{[-1,1]}|f|+2 \max _{[-1,1]}\left|f^{\prime}\right|, \quad f \in C^{\prime} .
$$

In particular, $\left|e^{i t T}\right| \leqq 1+2|t|=O(|t|)$.

However the projection associated with the isolated point $1 / n$ of the spectrum is

$$
E\left(\frac{1}{n}\right)=0 \oplus \cdots \oplus E_{n} \oplus 0 \oplus \cdots \quad(n= \pm 1, \pm 2, \cdots)
$$

which has norm $\geqq|n|$. Since the projections $E(1 / n)$ are not uniformly bounded, $T$ is not spectral and therefore $D \neq X$ (by Theorem 2.1). Let

$$
x=\left\{x_{n}\right\} \in X, \text { where } x_{n}=\left(x_{n 1}, x_{n 2}\right) \in H_{n} .
$$

It follows easily from (1) that $x \in D$ whenever $\sum_{n=1}^{\infty} n^{2}\left|x_{n 2}\right|^{2}<\infty$ (in particular, vectors of the form $\left\{x_{1}, \cdots, x_{n}, 0,0, \cdots\right\}$ are in $D$, hence $D$ is dense in $X$ ). For vectors $x$ with the latter property, we have a decomposition $T x=S x+N x$ as in Theorem 2.1, in which $N=0$ and

$$
E(\cdot) x=\sum_{n=-\infty}^{\infty} \delta_{1 / n}(\cdot) E\left(\frac{1}{n}\right) x
$$

where $\delta_{s}(\cdot)$ denotes the delta measure at $s \in \boldsymbol{R}$.

Let $K_{n}$ be a two-dimensional Hilbert space with the norm $\|x\|=\left\{\left|x_{n 1}\right|^{2}\right.$ $\left.+n^{2}\left|x_{n 2}\right|^{2}\right\}^{1 / 2}$, and let $K=\sum_{n=1}^{\infty} \oplus K_{n}$. Then $K \subset D, K$ is densely contained in $X$ and is invariant under $T$. Moreover, $T \mid K$ is continuous as an operator in $K$, and the conclusion of Theorem 2.14 is valid with $Y$ replaced by $K$. Since $K$ is reflexive (as a Hilbert space), $T \mid K$ is spectral of type 1 (in fact, it is spectral of scalar type, and its resolution of the identity is $E(\cdot) \mid K$ for $E(\cdot)$ as in (3)).

3.2. We consider an example in which $\sigma(T)$ may be a perfect set.

Let $F$ be an arbitrary uncountable closed subset of $(0,1]$. We shall exhibit an operator $T$ on a suitable Banach space $X$, with the following properties:

(a) $T$ satisfies (1.1) with $k=1$,

(b) $\sigma(T)=F$, and

(c) $D$ is dense in $X$, but $D \neq X$.

By $§ 1$, it suffices to find a nonspectral operator $T$ satisfying (a) and (b), whose eigenvectors are fundamental in $X$. 
First, define $T_{0}$ on $L^{p}[0,1](1 \leqq p<\infty)$ by $\left(T_{0} \phi\right)(x)=x \phi(x)+\int_{x}^{1} \phi(t) d t$, $\phi \in L^{p}[0,1] ; x \in[0,1] . T_{0}$ is of class $C^{\prime}:$ its $C^{\prime}$-operational calculus is given by $f \rightarrow T_{0}(f)$ where

$$
\left[T_{0}(f) \phi\right](x)=f(x) \phi(x)+\int_{x}^{1} f^{\prime}(t) \phi(t) d t, \quad \phi \in L^{p}[0,1], \quad x \in[0,1], \quad f \in C^{\prime} .
$$

We have $\left|T_{0}(f)\right| \leqq|f|_{1, T_{0}}$, and in particular

$$
\left|e^{i t T_{0}}\right|=\left|T_{0}\left(e^{i t s}\right)\right| \leqq 1+|t|=O(|t|) \text { for } t \in R
$$

The spectrum of $T_{0}$ is equal to $[0,1]$. The characteristic function $c_{s}$ of the interval $[0, s](0<s \leqq 1)$ is an eigenvector corresponding to the eigenvalue $s$. Let $X$ be the closed linear span in $L_{p}[0,1]$ of all the functions $c_{s}$ with $s \in F$. $X$ is a closed invariant subspace for $T_{0}$. Let $T=T_{0} \mid X$. The spectrum of $T$ is pure point and is equal to $F$; moreover, the eigenvectors of $T$ are fundamental in $X$. If $T$ was spectral, it would be of type 1 (since $T$ is of class $C^{\prime}$ ). Then, by Theorem 1, p. 56 in [4], its point spectrum would be at most countable, contrary to our hypothesis on $F$. Hence, $T$ is not spectral, and we conclude that it has properties (a), (b), (c).

Let $W \subset D$ be the linear span of all $c_{s}$ with $s \in F$. For each $\Delta \in \mathscr{B}$, let $E(\Delta) \in T(W)$ be defined by

$$
E(\Delta) c_{s}=c_{s} \delta_{s}(\Delta), \quad s \in F
$$

Since the vectors $c_{s}$ are linearly independent, $E(\Delta)$ is well defined on $W$. Clearly, $E(\cdot)$ is a generalized spectral measure on $W$, supported by $F=\sigma(T)$ and commuting with $T$. Moreover, if $p$ is any polynomial and $\phi \in W$ (say $\left.\phi=\sum_{j=1}^{n} \alpha_{j} c_{s_{j}}, \alpha_{j} \in C, s_{j} \in F\right)$, then

$$
p(T) \phi=\sum_{j=1}^{n} \alpha_{j} p\left(s_{j}\right) c_{s_{j}}=\int_{F} p(s) d_{s}\left\{\sum_{j=1}^{n} \alpha_{j} c_{s_{j}} \delta_{s_{j}}(s)\right\}=\int_{\sigma(T)} p(s) d E(s) \phi,
$$

where $E(\cdot)$ is given by (4).

Thus the conclusions of Theorem 2.1 are valid with $W$ in place of $D$ and with $N=0$. If $F$ is a Lebesgue null set and $1<p<\infty$, the uniqueness assertion in Theorem 2.1 implies that the nilpotent part of $T$ restricted to $W \subset D$ is zero, and the resolution of the identity of $T$ restricted to $W$ is given by (4).

\section{BIBLIOGRA PHY}

1. N. Dunford, Spectral operators, Pacific J. Math. 4 (1954), 321-354.

2. —_ A survey of the theory of spectral operators, Bull. Amer. Math. Soc. 64 (1958), 217-274.

3. N. Dunford and J. Schwartz, Linear operators, Parts I, II, Interscience, New York, 1958; 1964.

4. S. R. Foguel, The relations between a spectral operator and its scalar part, Pacific J. Math. 8 (1958), 51-65. 
5. C. Foras, Une application des distributions vectorielles à la théorie spectrale, Bull. Sci. Math. (2) 84 (1960), 147-158.

6. S. Kantorovitz, On the characterization of spectral operators, Trans. Amer. Math. Soc. 111 (1964), 152-181.

7. - Classification of operators by means of their operational calculus, Trans. Amer. Math. Soc. 115 (1965), 194-224.

8. M. A. Naimark, Normed rings, Noordhoff, Groningen, 1959.

9. E. Nelson, A functional calculus using singular Laplace integrals, Trans. Amer. Math. Soc. 88 (1958), 400-413.

10. L. Schwartz, Théorie des distributions. I, rev. ed., Hermann, Paris, 1957.

INSTITUTE FOR ADVANCED STUDY,

Princeton, New Jersey

YALE UNIVERSITY,

New Haven, Connecticut 